\title{
Juvenile granulosa cell tumour: a rare clinical entity
}

\author{
Kaliki Hymavathi Reddy*, Pavithra D, Ragini R, Pallavi S
}

Department of Obstetrics \& Gynaecology, Narayana Medical College \& Hospital, Nellore-524002, A.P., India

Received: 8 October 2014

Accepted: 1 November 2014

\section{*Correspondence:}

Dr. Kaliki Hymavathi Reddy,

E-mail: drhymakrreddy@yahoo.co.in

Copyright: (C) the author(s), publisher and licensee Medip Academy. This is an open-access article distributed under the terms of the Creative Commons Attribution Non-Commercial License, which permits unrestricted non-commercial use, distribution, and reproduction in any medium, provided the original work is properly cited.

\begin{abstract}
Ovarian cancer is the third most common neoplasm of the female genital tract. Based on the cell type of origin, primary ovarian malignancies are classified into surface epithelium, germ cell, and sex cord tumors. Sex cord tumors account for $1 \%$ to $2 \%$ of ovarian malignancies. They may contain granulosa cells, theca cells, sertoli cells, or fibroblasts of gonadal stromal origin. Granulosa Cell Tumours (GCTs) account for approximately 2-5\% of all ovarian tumors and can be divided into adult $(95 \%)$ and juvenile $(5 \%)$ types based on histologic findings. GCTs secrete estrogen thus resulting in menstrual irregularities in the affected individual. More serious estrogen effects can occur in various end organs such as uterus resulting in endometrial hyperplasia, endometrial adenocarcinomas and increased risk of breast cancers. Androgen production is also reported but rare and produces virilization in the affected women. Juvenile Granulosa Cell Tumours (JGCTs) are clinically \& histopathologically distinct from the GCTs. They are rarely encountered but mostly in youngsters. Surgery is the primary modality of treatment with chemotherapy being reserved for advanced or recurrent disease states. We herewith report an interesting case of JGCT in a young teenage girl.
\end{abstract}

Keywords: Ovarian sex cord stromal tumours, Juvenile granulosa cell tumour

\section{INTRODUCTION}

Granulosa Cell Tumours (GCTs) account for approximately $2-5 \%$ of all ovarian tumors and can be divided into adult $(95 \%)$ and juvenile $(5 \%)$ types based on histologic findings. Juvenile Granulosa Cell Tumors (JGCTs) are rare sex-cord stromal tumours occurring in the younger age groups. JGCT is clinically \& histopathologically distinct from the GCTs. We here with report an interesting case of JGCT in a young girl.

\section{CASE REPORT}

A 17 year old unmarried girl, came with complaints of irregular cycles for three months (3-5/35-45d) followed by amenorrhoea of 6 months duration. She is a known hypothyroid since 3 years \& on treatment. Attained menarche at 14 years, with regular cycles (3-5/28-30d) for 2 years.

\section{On clinical examination}

General condition good. BMI $=30 \mathrm{Kg} / \mathrm{m}^{2}$. Vitals: Within Normal Limits (WNL). Heart and lungs clinically normal. Secondary sexual characters well developed.

\section{Abdominal examination}

A non-tender mass of 18 weeks size occupying the lower abdomen, firm in consistency, mobile from side to side \& lower border of the mass could not be made out.

\section{Vaginal examination}

Not attempted. 


\section{Per rectal examination}

Vague fullness present in pouch of Douglas. Uterus could not be felt separately. Rectal mucosa free.

\section{Investigations}

Baseline investigations: WNL. Thyroid function tests\& serum prolactin: normal.

\section{Tumour markers}

Serum CA-125, CEA, AFP, $\beta$-hCG \& LDH were WNL.

\section{Ultrasound}

Large $11.2 \times 6.2 \mathrm{~cm}$ sized, well defined mass, predominantly cystic with increased vascularity is noted in the hypogastric region?arising from left ovary. No calcification or solid component noted. Uterus normal in size and echotexture. Right ovary visualised, normal. Liver, spleen, gall bladder and kidneys were normal. No e/o of free fluid in peritoneal cavity.

\section{Chest X-ray}

Normal.

\section{CECT}

Uterus normal in size and attenuation. Right ovary is normal in size and attenuation. Well defined heterogeneously enhancing mass in the left lower abdomen and pelvis. Mild left hydroureteronephrosis present. No e/o free fluid noted in the peritoneal cavity. Impression: left ovarian mass.

\section{Doppler study}

No obvious e/o of neovascularisation.

\section{Management}

Patient posted for exploratory laparotomy. Intraoperatively: There was no free fluid in the peritoneal cavity. Fluid with saline wash collected for cytology negative. No macroscopic omental deposits noted. Under surface of liver and diaphragm were normal. No e/o of enlarged para aortic lymph nodes. Uterus was normal in size, right ovary slightly enlarged \& cystic. Tumour arising from left ovary $(20 \times 15 \mathrm{~cm})$ with variable consistency. Capsule intact.

Ipsilateral tube could not be saved as it was merged with tumour capsule therefore left salpingo-oopherectomy was done with removal of tumour intoto with intact capsule. Multiple omental \& peritoneal biopsies were taken. Postoperative period uneventful.

\section{Histopathology report}

Juvenile granulose cell tumour (well differentiated). Omental \& peritoneal bits negative for malignancy

\section{Immunohistochemistry}

\section{Vimentin positive}

In view of the HP report patient was referred to oncologist for opinion and further management. Oncologists opined that since it is a stage1A tumour there is no need for any further chemotherapy, but she has to be under follow up.

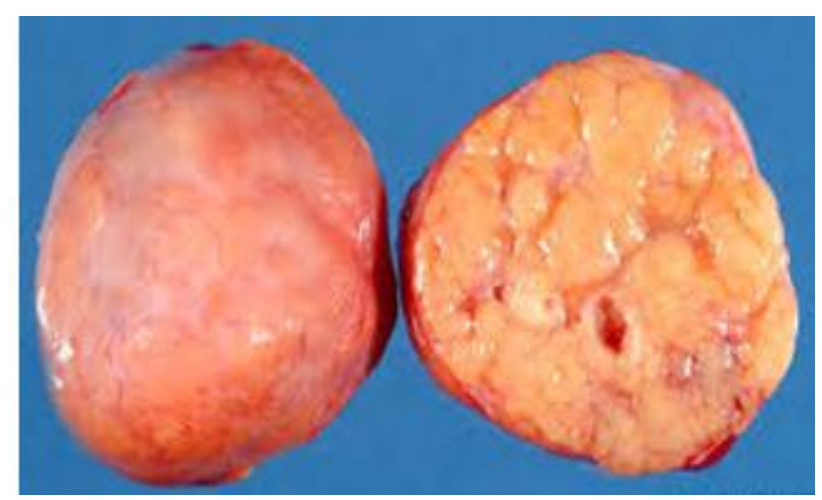

Figure 1: JGCT-Macroscopic appearance.

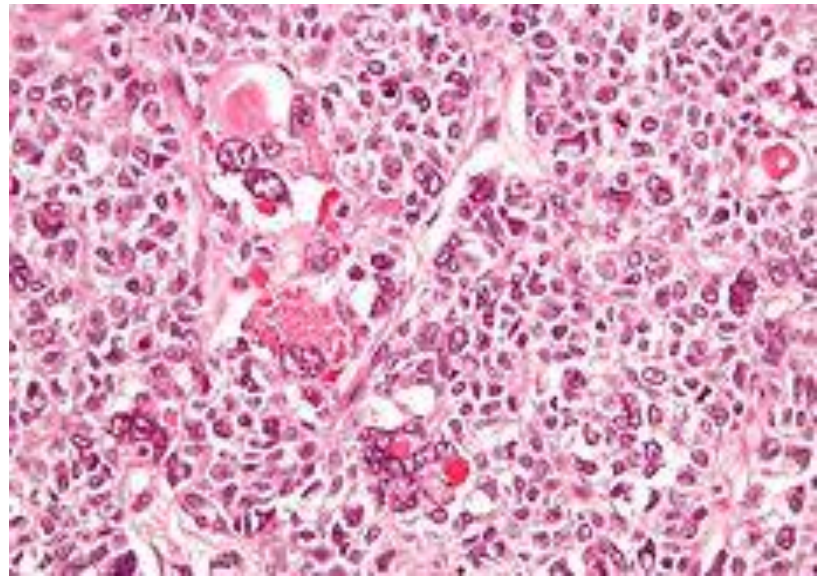

Figure 2: H\&E stain-JGCT with hyaline globules.

\section{DISCUSSION}

Ovarian cancer is the third most common neoplasm of the female genital tract. Based on the cell type of origin, primary ovarian malignancies are classified into surface epithelium, germ cell, and sex cord tumors. Sex cord tumors account for 1 to $2 \%$ of ovarian malignancies. They may contain granulosa cells, theca cells, sertoli cells, or fibroblasts of gonadal stromal origin. Approximately $70 \%$ of sex cord tumors are Granulosa Cell Tumours (GCT). Granulosa cell tumors have bimodal age distribution but the peak incidence is in postmenopausal period with median age of diagnosis 
around 50-55 years. ${ }^{1}$ Juvenile Granulosa Cell Tumors (JGCTs) are rare sex-cord stromal tumours occurring in the younger age groups. GCTs were described for the first time in 1855 by Rokitansky. These tumors are malignancies with a relatively favorable prognosis. They are characterized by a prolonged natural history and a tendency to late recurrences. ${ }^{2}$

Kalfa et al. identified a mutation FOXL2 (transcription factor gene) in the majority of GCTs, particularly in adult form. This FOXL2 could be the next target for use in treatment. Yoo et al. also identified mutations of genes Fas, FLIP and Bcl-2 related to alterations of apoptosis. Genetic mutations R201C and R201H were exclusively localized in them. ${ }^{3}$

GCT's secrete estrogen thus resulting in menstrual irregularities and amenorrhoea in the affected individuals. A more serious estrogen effects can occur in various endorgans such as Uterus resulting in endometrial hyperplasia, endometrial adenocarcinomas and increased risk of breast cancers. Irregular cycles and amenorrhoea in our case can be attributed to a state of hyperestrogenism caused by the tumour secretions.

Inhibin is a peptide hormone produced by ovarian granulosa cells that has a regulatory effect on Follicular Stimulating Hormone (FSH) secretion. Increased serum inhibin and subsequent FSH suppression have been proposed as causative factors for amenorrhea. ${ }^{4}$ This can also be another explanation for amenorrhoea in our case, attributed to the inhibin induced FSH suppression effect.

Prepubertal patients afflicted with secretary JGCTs present with clinical evidence of precocious pseudopuberty ${ }^{5}$ including breast enlargement, development of pubic and axillary hair, vaginal secretions, irregular uterine bleeding, somatoskeletal changes, and other secondary sexual characteristics. Androgen production is rare and produces virilization in women. These tumors are usually solid or predominantly solid and only few cases of predominantly cystic JGCT have been reported. Even elevated levels of serum Testosterone reported in some cases. ${ }^{6}$

In the indexed case she attained menarche normally at age of 14 years \& her secondary sexual characters are normal. Her irregular oligomenorrhoeic cycles for three months followed by six months of amenorrhoea may suggest androgen secretion of the tumour as mentioned but her serum Testosterone levels were within normal range.

GCTs are best considered as unusual indolent neoplasm of low malignant potential with late recurrences. The optimal management of sex cord ovarian tumors is limited by their low incidence, the multiplicity of their histologic patterns, and their variable biologic behaviour.

As for tumor markers, the measurement of serum AFP and $\beta$-hCG level is important to exclude secreting germ cell tumours ${ }^{7}$ which were normal in the index patient. Patients with both adult and juvenile types of GCTs have been shown to have increased levels of inhibin which may return to the baseline levels postoperatively. Among all clinical parameters, tumor stage is of greatest prognostic value and must be carefully assigned for all patients. Of the various features that were evaluated for prognostic significance, a low stage of the tumor correlated best with survival. Although there was a relation of size, mitotic activity, and nuclear atypia to the outcome when tumors of all stages were evaluated, no such relation was evident when only stage Ia and Ib tumors were considered.

Imaging characteristics of adult and juvenile granulosa cell tumors are non-specific ${ }^{8}$ and these tumors cannot be reliably distinguished from other ovarian neoplasms on imaging alone. On cross-sectional CT imaging and sonography, their appearance vary widely, but they often appear as a single large multiloculated cystic mass with solid components. They have multiple septations which can be thin, or thick and irregular. The adult form shows more variability with regards to the cystic component and can occasionally appear predominantly solid. Intratumoral haemorrhage, central areas of necrosis and fibrous degeneration can result in a heterogeneous solid appearance MR imaging is more distinctive. T1W MR images can demonstrate intracystic high signal suggesting characteristic intratumoral haemorrhage present in up to $71 \%$ of cases.

On T2W images, tumors have a sponge-like appearance, indicating alternating solid and cystic spaces. Tumoral secretion of estrogen can cause uterine enlargement and endometrial thickening, which can be additional imaging findings in $50 \%$ of patients. $10 \%$ of adult type granulosa cell tumors are associated with endometrial adenocarcinoma, thus some investigators have suggested preoperative endometrial biopsy in these patients. Metastasis, though rare at initial presentation, appear as cystic liver masses or peritoneal implants, similar to epithelial ovarian neoplasms. MRI is not done in our case for some reasons.

Patients with JGCT typically present at an early stage I and enjoy a favorable prognosis \& five year survival of $95-100 \%$ if diagnosed at early stage. Recurrences are uncommon and typically occur within the first year. The incidence of this group of tumour is same throughout the world. Majority of JGCTs present as localized disease confined to the ovary, and usually behave in a benign manner despite having histopathological features of malignancy. However, those with more advanced-stage disease (FIGO stages II, III, or IV) may experience an aggressive clinical course with a short remission to relapse or death. Both juvenile and adult type GCTs are almost always unilateral. As per the unilateral origin of the tumor with intact capsule \& negative peritoneal cytology, our case was considered as stage 1A. 
JGCT is distinguished from AGCT by 1) more commonly occurring in young women (<30 years of age), 2) having follicles that are more irregular in shape and size, 3) lacking Call-Exner bodies, 4) having rounder nuclei that lack nuclear grooves, 5) typically having more abundant eosinophilic to vacuolated cytoplasm and 6) having follicles containing basophilic secretions. The histological report of our patient was consistent with the above mentioned JGCT features.

Though immunohistochemical staining for inhibin and smooth muscle Actin is positive in almost all cases, these tests could not be done in our patient due to specific problems like lack of reagents which could have given more distinctive information of JGCT and also help in monitoring for relapse in some cases. Vimentin positivity though not specific for the diagnosis of JGCT it is found to be helpful in distinguishing well differentiated GCT's from poorly differentiated ones. As per this the histopathology report in our case was given as "well differentiated juvenile granulosa cell tumour".

Various modalities of treatments have been used ranging from surgical removal of the tumour to chemotherapy, radiotherapy and other treatments. In adolescent girls and young adult females, fertility sparing surgery can be done. The role of post-operative chemo- or radiotherapy in stage I disease and those with completely resected tumor has not been defined. Nevertheless, the use of adjuvant chemotherapy or radiotherapy has sometimes been associated with prolonged disease-free survival and possibly overall survival. Some have used platinum agent as standard treatment, either combined with Vinblastine and bleomycin or adriamycin and cyclophosphomide. Other authors now recommend the use of Bleomycin, Eptoposide and Platinum (BEP). ${ }^{9}$ Studies suggested that patients aged 19 years or below and with early stage disease should have only surgery and close monitoring for tumour recurrence.

Others have used stem cell transplantation. Newer agents that block angiogenesis are being studied, two are being tried currently: sunitinib and bevacizumab. Hormone based treatments like Paclitaxel and taxane in combination with platinum are also being advocated. Taxanes demonstrated activity against sex cord-stromal tumors of the ovary and may be less toxic than BEP. Taxane and platinum combination chemotherapy warrants further investigation in this disease. ${ }^{10}$

The use of radiotherapy has not been shown to confer any survival benefit to JGCT at any stage. Finally, surgery continues to be the primary corner stone of initial treatment with chemotherapy being reserved for advanced or recurrent disease states. Surgery is modified to unilateral salpingoophorectomy to preserve fertility in the child-bearing women once extra-ovarian spread has carefully been ruled out. These tumors are often not suspected until frozen sections are made, and since frozen sections are fraught with many inaccuracies, their diagnosis requires a great deal of expertise. In our case also since she is only 17 years and the tumour staging being IA, the Oncologists opined that following primary surgery there is no need for further chemotherapy but she needs to be followed up. It is now one year after surgery $\&$ she is under close follow up with the gynaecologist \& oncologist. She is doing fine at present and as per the oncologist's opinion she has to be under close follow up for atleast three years as in contrast to AGCT, recurrences in patients with JGCT typically occur within the first three years.

\section{CONCLUSIONS}

Juvenile granulosa cell tumour is a distinctly rare tumour encountered in the clinical practice. As multifaceted clinical presentations coupled with the unpredictable biological behaviour with late relapses are the pitfalls necessitating a high degree of suspicion for accurate clinical and pathological diagnosis as well as management. Surgery is the primary modality of treatment in the early stage disease.

\section{Funding: No funding sources \\ Conflict of interest: None declared \\ Ethical approval: Not required}

\section{REFERENCES}

1. Divya Khosla, Kislay Dimri, Romeeta Trehan. Ovarian granulosa cell tumor: clinical features, treatment, outcome \& prognostic factors. N Am J Med Sci. 2014 Mar;6(3):134-8.

2. Sakina Sekkate, Mouna Kairouani, Badr Serji, Adnane Tazi, Hind Mrabti, Saber Boutayeb, et al. Ovarian granulosa cell tumors: a retrospective study of 27 cases and a review of the literature. World $\mathbf{J}$ Surg Oncol. 2013;11:142.

3. Mava Y, Chinda JY, Alhaji MA, Nggada HA. Childhood ovarian juvenile granulosa cell tumour: a case report and review of literature. Niger J Paediatr. 2012:39(4):199-201.

4. Adachi T, Sato H, Nakayama S, Shimizu S, Matsui $H$, Nakabayashi M. Primary amenorrhea due to juvenile granulosacell tumor of the ovary: a case report. J Obstet Gynaecol Res. 2012;38(3):597-6.

5. Nisha Nigil Haroon, Gaurav Agarwal, Rakesh Pandey, Preeti Dabadghao. Juvenile granulosa cell tumor presenting as isosexual precocious puberty: A case report and review of literature. Indian $\mathbf{J}$ Endocrinol Metab. 2013 Jan-Feb;17(1):157-9.

6. Nomelini RS, Micheletti AM, Adad SJ, Murta EF. Androgenic juvenile granulosa cell tumor of the ovary with cystic presentation: a case report. Eur $\mathbf{J}$ Gynaecol Oncol. 2007;28(3):236-8.

7. Colombo, Parma G, Zanagnolo V, Insinga A. Management of ovarian stromal cell tumors. J Clin Oncol. 2007;25(20):2944-51.

8. Kristin RR, William M. An unusual case of juvenile granulose cell tumour of the ovary. Radiol Case Rep. 2009;4:178. 
9. Pectasides D, Pectasides E, Psyrri A. Granulosa cell tumor of the ovary. Cancer Treat Rev. 2008 Feb;34(1):1-12.

10. Brown J, Shvartsman HS, Deavers MT, Ramondetta LM, Burke TW, Munsell MF, et al. The activity of taxanes compared with bleomycin, etoposide, and cisplatin in the treatment of sex cord-stromal ovarian tumors. Gynaecol Oncol. 2005;97(2):489-96.

DOI: $10.5455 / 2320-1770 . i j \operatorname{cog} 20141259$

Cite this article as: Reddy $\mathrm{KH}$, Pavithra D, Ragini R, Pallavi S. Juvenile granulosa cell tumour: a rare clinical entity. Int J Reprod Contracept Obstet Gynecol 2014;3:1150-4. 